\title{
An ALMA and ATCA Molecular Line Survey Toward Centaurus A
}

\author{
Jürgen Ott ${ }^{1}$, Mark McCoy ${ }^{2}$, David Meier ${ }^{2}$, et al. \\ ${ }^{1}$ National Radio Astronomy Observatory, \\ 1003 Lopezville Road, Socorro, NM 87801, USA \\ email: jott@nrao.edu \\ ${ }^{2}$ New Mexico Institute of Mining and Technology, Physics Department, \\ 801 Leroy Place, Socorro, NM 87801, USA \\ email: mccoy@nmt.edu; dmeier@nmt.edu
}

\begin{abstract}
We present Atacama Large Millimeter/submillimeter Array (ALMA) and Australia Telescope Compact Array (ATCA) data of molecular absorption lines toward the bright central core of the prominent, nearby $(\mathrm{d} \sim 3.8 \mathrm{Mpc})$ radio galaxy Centaurus $\mathrm{A}$, at $13,7,3$, and $1 \mathrm{~mm}$ wavelengths. The line of sight crosses the prominent dust lane and continues through the disk and eventually through gas that may be very close to the central supermassive black hole. The goal of our survey is to determine the physical conditions of the gas via analyses of molecular line tracers including molecular abundances and excitation conditions that are sensitive to changes in temperature, density, ionization, and shocks. This study allows us to derive the physical processes that are shaping each molecular environment.
\end{abstract}

Keywords. ISM: molecules — galaxies: individual (Centaurus A) — galaxies: ISM

Fig. 1 displays a small selection of preliminary, first spectra. The $\mathrm{HCO}^{+}(1-0)$ provides a good representation of the overall structure: very narrow, deep absorption features near the systemic velocity of Cen A $\left(\sim 550 \mathrm{~km} \mathrm{~s}^{-1}\right)$, as well as a broad component at higher velocities that exhibits superimposed narrow components. The molecular spectra trace the chemistry and physical conditions of each component. Surprisingly, the CO isotopologues are very weak in the broad component, and might be dissociated in this particular gas phase. Poly-atomic molecules seem to have stronger absorption in the broad component relative to the narrow components. This indicates that the narrow and broad components trace very different molecular environments. Evidently the dynamically active, broad absorption component comes from a more extreme, denser environment that is embedded in a strong radiation field. This gas is likely located closer to the nucleus of Cen A and may indicate outflowing or infalling material from/to the black hole.
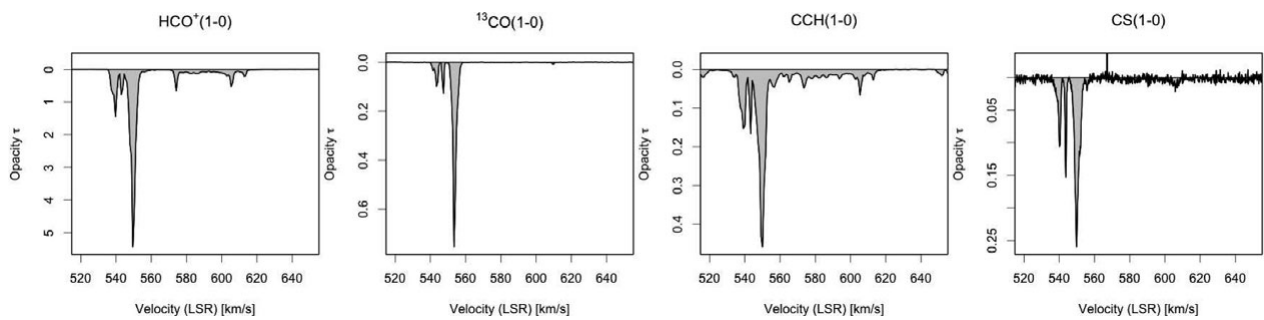

Figure 1. First example spectra of our Cen A ALMA $\left(\mathrm{HCO}^{+},{ }^{13} \mathrm{CO}, \mathrm{CCH}\right)$ and ATCA (CS) survey. Narrow components are close to the systemic velocity of Cen A. 